\title{
Optimierung der pharmakologischen Langzeitbehandlung ersterkrankter schizophrener Patienten
}

\author{
Mathias Riesbeck', Martina von Wilmsdorff', Robert Krohmer' ${ }^{1}$, Wolfgang Wölwer' ${ }^{1}$, Ronald Bottlender ${ }^{2}$, Hans-Jürgen Möller ${ }^{2}$, \\ Wolfgang Gaebel ${ }^{1}$ \\ ${ }^{1}$ Klinik und Poliklinik für Psychiatrie und Psychotherapie der Heinrich-Heine-Universität Düsseldorf - Rheinische Kliniken Düsseldorf \\ 2Klinik für Psychiatrie und Psychotherapie der Ludwig-Maximilians-Universität München
}

psychoneuro 2004; 30 (10): 615-619

$\mathrm{N}$ ach Abschluss der Akutbehandlung einer schizophrenen Erstmanifestation mit Suppression und Remission der (Positiv-) Symptomatik gilt es in der Langzeitbehandlung die Remission weiter zu stabilisieren und auf andere (Negativ-) Symptome auszuweiten, das Funktionsniveau des Patienten allgemein wieder größtmöglich herzustellen und v.a. Reexazerbationen zu verhindern (vgl. 1, 5). Hierfür stehen mittlerweile eine Reihe effektiver bio-psycho-sozialer Behandlungsansätze zur Verfügung, die Eingang in verschiedene Behandlungsleitlinien basierend auf empirischer Evidenz und Expertenkonsens gefunden haben (z.B. 1, 2, 5, $12,13,17-19)$. Dennoch sind wichtige Aspekte der Langzeitbehandlung noch ungeklärt und bedürfen weiterer empirischer Forschung.

Eine davon betrifft die Frage, ob atypische gegenüber typischen Neuroleptika hinsichtlich der rezidivprophylaktischen Wirksamkeit überlegen sind, wie bereits für mehrfacherkrankte Patienten in der Akut(14) und in der Langzeitbehandlung $(3,15)$ nachgewiesen wurde. Die Neuroleptika der „Zweiten Generation" weisen ein günstigeres Wirkungs-Nebenwirkungsprofil auf, was sich u.a. durch eine bessere Wirkung auf Negativsymptome bei reduzierten extrapyramidalen Nebenwirkungen bemerkbar macht und zu einer

In den letzten Jahrzehnten sind eine Reihe bio-psycho-sozialer Behandlungsstrategien entstanden, die eine effektive Langzeitbehandlung von Patienten mit schizophrenen Störungen ermöglichen und die es durch weitere Forschung zu optimieren gilt. Eine multizentrische Verbundstudie im Rahmen des Kompetenznetzes Schizophrenie versucht dies speziell für schizophrene Erstmanifestationen, indem u.a. zwei Hauptfragestellungen untersucht werden: Gibt es Unterschiede in der Rezidivrate a) der postakuten einjährigen Erhaltungsmedikation mit einem niedrigdosierten typischem vs. atypischen Neuroleptikum und b) anschließendem Fortführen vs. Absetzen der neuroleptischen Medikation, jeweils ergänzt durch prodromgestützte Frühintervention. Erste Ergebnisse der noch laufenden Studie sprechen für eine rezidivprophylaktische Effektivität beider Neuroleptika bei einer hohen Abbruchquote. Entsprechend gilt es spezielle Programme zu entwickeln, um (ersterkrankte) schizophrene Patienten in der effektiven Behandlung zu halten.

verbesserten Compliance beiträgt. Allerdings gilt zu berücksichtigen, dass bei diesem Vergleich die typischen Neuroleptika meist in einer höheren Dosierung verabreicht wurden als die Atypika. Entsprechende metaanalytische Vergleiche von niedrigdosierten typischen Neuroleptika mit dosisäquivalenten Atypika ergaben dagegen keine Unterschiede in der Symptomreduktion und Akzeptanz (10) bzw. in dem Ausmaß extrapyramidaler Nebenwirkungen (16), wohingegen andere Autoren (4) weiterhin Unterschiede zu Gunsten der Atypika nachweisen konnten. Zudem werden neuerdings kritisch die unerwünschten Wirkungen der Atypika hinsichtlich Gewichtszunahme und metabolischer Effekte (Induktion von Typ-II-Diabetes, erhöhte Lipidwerte) mit ihrem Risikopotenzial für kardiovaskuläre Erkrankungen diskutiert.
Auch für ersterkrankte Patienten werden mittlerweile die atypischen Neuroleptika als die zu präferierende medikamentöse Behandlung empfohlen (z.B. von dem britischen National Institute of Clinical Excellence, 19), wenngleich empirische Evidenz für diese Empfehlung bis dato lediglich für die Akutbehandlung vorliegt $(6,20)$. Empirische Daten, ob die für Mehrfacherkrankte gefundene rezidivprophylaktische Überlegenheit in der Langzeitbehandlung (Rückfallraten von $15 \%$ vs. $23 \%$; 15 ) auch für ersterkrankte Patienten gilt, stehen weiterhin aus.

Ungeklärt ist weiterhin die indizierte Dauer der neuroleptischen Therapie bei Patienten nach der ersten schizophrenen Episode. Gängige Behandlungsleitlinien (z.B. 1, 5) empfehlen eine mindestens einjährige Erhaltungsmedikation nach abgeschlossener Akuttherapie der 


\section{Abb. 1 Studien-Design und kooperierende Projekte}

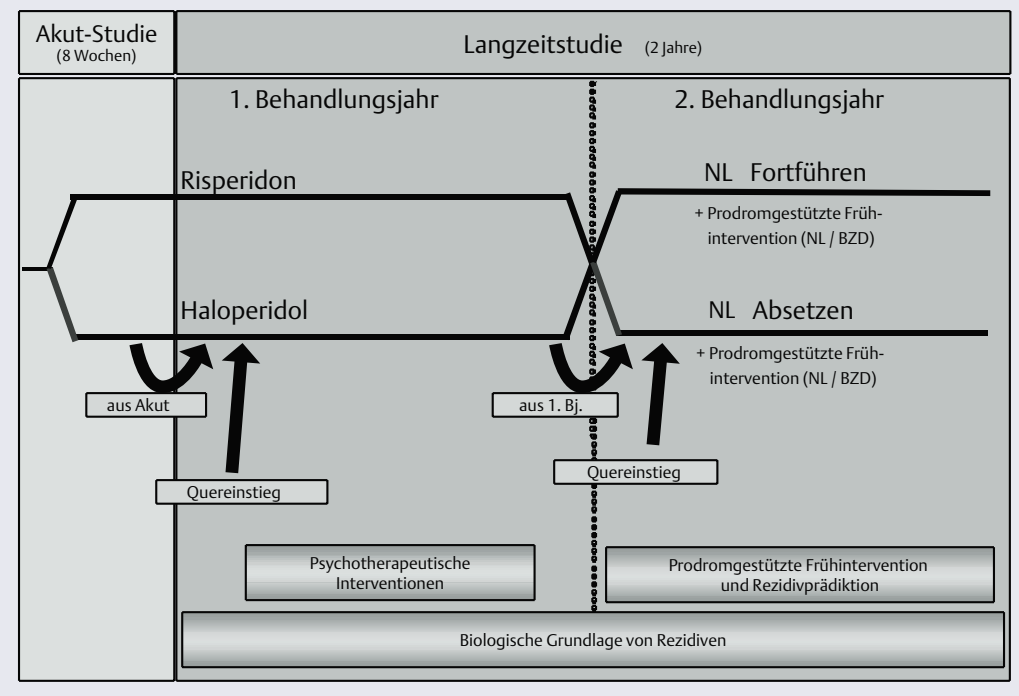

Erstmanifestation; wie anschließend remittierte, soweit stabile Patienten weiterbehandelt werden sollen ist offen. Eigene post hoc Analysen einer früheren Studie ergaben keine signifikanten Unterschiede in der Rückfallrate bei ersterkrankten Patienten unter Erhaltungsmedikation im Vergleich mit Intervalltherapie, wohingegen die Rezidivrate von Mehrfacherkrankten unter Intervalltherapie deutlich höher war als unter Dauer- (Erhaltungs-)therapie (7). Diese Befunde lassen ein schrittweises Absetzen der neuroleptischen Medikation nach einem Jahr bei ersterkrankten Patienten auch unter gen möglich erscheinen. werden: don) Neuroleptikum? rezidivprophylaktischen Erwägun-

Zur Klärung dieser beiden Fragen will die 2-jährige „Langzeitstudie“ innerhalb des Kompetenznetzes Schizophrenie einen empirischen Beitrag leisten indem folgende Hauptfragestellungen untersucht

- im 1. Behandlungsjahr: Gibt es Unterschiede in der Rezidivrate bei ersterkrankten schizophrenen Patienten unter Erhaltungstherapie mit einem niedrigdosierten typischen (Haloperidol) vs. atypischen (Risperi-

\section{Abb. 2 Symptomverlauf und Funktionsniveau} im ersten Behandlungsjahr ( $n=115$; LOCF-Analyse)

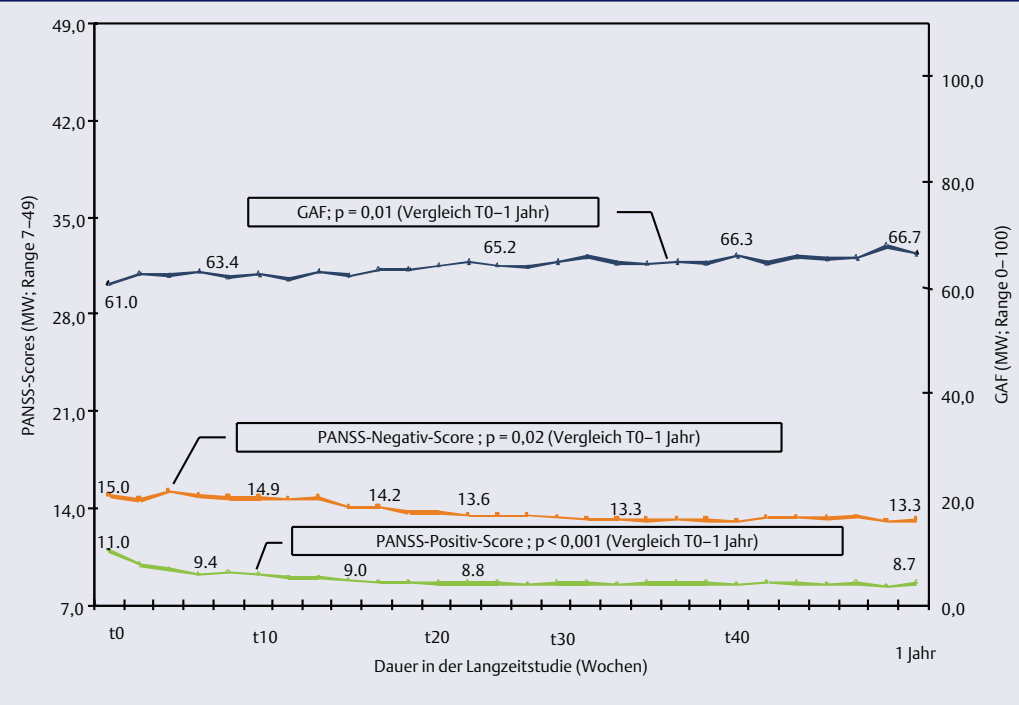

- im 2. Behandlungsjahr: Unterscheidet sich die Rückfallrate bei bis dato stabil gebliebenen Patienten, wenn die neuroleptische Therapie weiter fortgeführt wird vs. schrittweise abgesetzt wird, in beiden Fällen ergänzt durch eine prodromgestützte Frühintervention.

Darüber hinaus werden weitere Aspekte der verschiedenen Langzeitbehandlungstrategien (explorativ) untersucht, wie z.B. differentielle Effekte der jeweiligen Interventionen auf Negativsymptomatik, kognitive Funktionen, Nebenwirkungen, Compliance oder Lebensqualität.

Zudem werden neben den pharmakotherapeutischen Fragestellungen in kooperierenden Projekten weitere Aspekte der Langzeitbehandlung und des Langzeitverlaufs untersucht. Eine Tübinger Arbeitsgruppe (Leitung PD Dr. Klingberg) prüft die (zusätzliche) rezidivprophylaktische Effektivität zweier psychotherapeutischer Interventionen (8-wöchige Psychoedukation im Vergleich mit einer 12-monatigen kognitiven Verhaltenstherapie), eine Düsseldorfer Arbeitsgruppe (Leitung Prof. W. Gaebel) analysiert den Verlauf von Prodromalsymptomen und anderer Parameter, um deren rezidivprädiktive Validität weiter zu verbessern, in enger Kooperation mit einer weiteren Düsseldorfer Arbeitsgruppe (Leitung Dr. W. Wölwer), die die biologische Grundlage von Rezidiven (Hirnmorphologie, neurofunktionale Korrelate etc.) untersucht, wie sie im ätiologisch zu Grunde liegenden Vulnerabilitäts-Stress-Coping-Modell hypothetisiert werden.

\section{Methodik}

Die Langzeitstudie wird im Verbund mit einer vorangehenden Akutstudie multizentrisch an 13 (universitären) psychiatrischen Kliniken durchgeführt (Bonn, Köln, Düsseldorf, München, Tübingen, Berlin, Essen, Jena, Mannheim, Göttingen, Mainz, Halle, Aachen; s.a. 9). Die randomisierte doppelblinde typische vs. atypische neuroleptische Behandlung wird nach Teilnahme an der Akutstudie in niedriger Dosis (mög- 
lichst zwischen 2 und $4 \mathrm{mg}$ Haloperidon/Risperidon) im ersten Behandlungsjahr weitergeführt (s. Abb. 1). Zudem können ersterkrankte Patienten ohne Teilnahme oder regulären Abschluss an der Akutstudie nach erfolgreicher Akutbehandlung in Form eines Quereinstieges in die Langzeitstudie eingeschlossen werden (mit initialer Randomisation und Verblindung). Zu Beginn des zweiten Behandlungsjahres werden die bis dato stabilen Patienten erneut randomisiert auf Fortführung ihrer neuroleptischen Behandlung oder deren schrittweises Absetzen (ebenfalls ergänzt durch die Möglichkeit eines Quereinstieges). In beiden Gruppen findet im Falle von frühen Anzeichen eines Rezidives eine medikamentöse Frühintervention statt (Neuroleptikum vs. Benzodiazepin; gleichfalls randomisiertes Design), gesteuert durch einen Entscheidungsalgorithmus, der im Vorfeld der Studie auf der Basis empirischer Befunde entwickelt wurde und der neben Prodromen auch andere rückfallprädiktive Parameter berücksichtigt (8).

An der Studie teilnehmen konnten alle Patienten der jeweiligen Zentren nach Erstmanifestation einer Schizophrenie (Diagnose F20.x nach ICD-10), mit einem Alter zwischen 18 und 55 Jahren, ausreichend deutschen Sprachkenntnissen und nach informierter Einwilligung. Ausschlusskriterien waren Schwangerschaft, Kontraindikation einer neuroleptischen Behandlung, deutliche Intelligenzminderung, hirnorganisch bedingte Beeinträchtigungen, Drogen-/Medikamentenmissbrauch oder -abhängigkeit sowie suizidales Verhalten in der Vorgeschichte.

Nach Einschluss in die Studie werden die Patienten 14-tägig gesehen und eine Reihe relevanter Informationen erhoben bezüglich (Skalenabkürzungen s. Tabelle 1): Psychopathologie (u.a. PANSS, CGI), Funktionsniveau (GAF), Nebenwirkungen (EPS, UKU, HAS, AIMS), Compliance (CRS), Einstellung gegenüber der neuroleptischen Behandlung (DAI), Prodromalsymptomen und Ausmaß psychosozialer Belastung (MEL). Darüber hinaus werden bei Studieneinschluss, nach einem Jahr und zu Studienabschluss Angaben erhoben zur Stress-

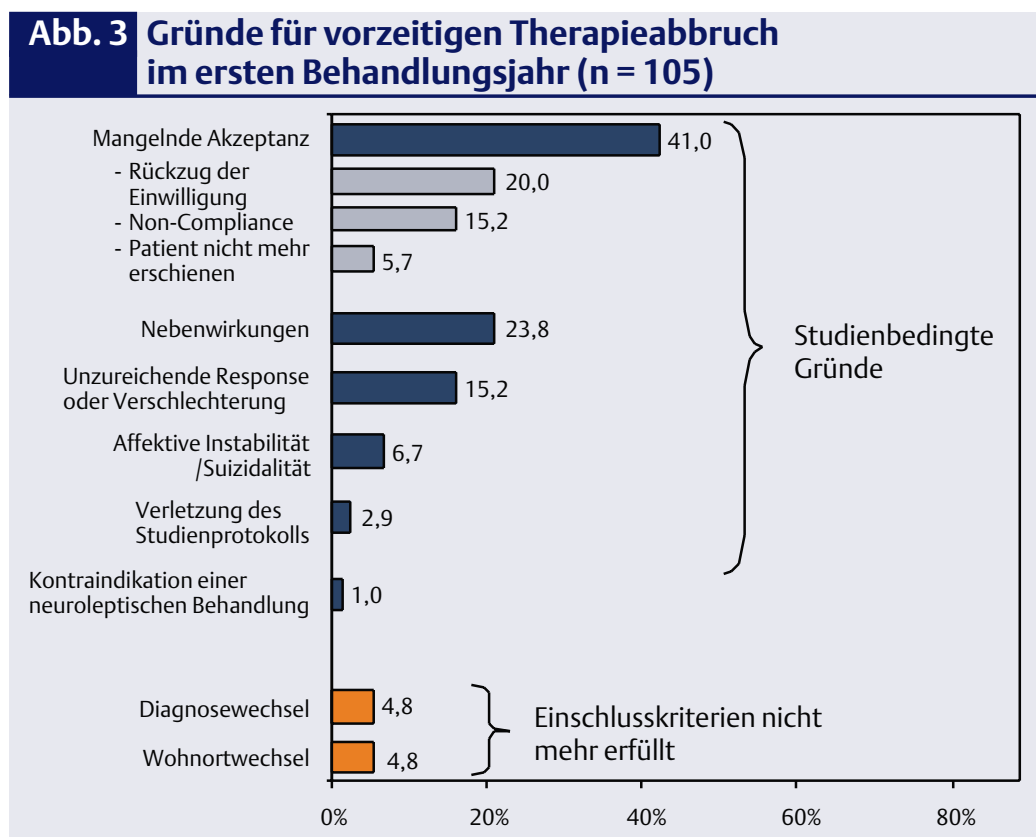

verarbeitung (SVF, FSKN), Familienatmosphäre (FEF), Lebensqualität (LQLP) sowie diverse neuromorphologische (CT), -funktionale (EEG, fMRT) und kognitive Maße (Gedächtnis, Aufmerksamkeit, visuomotorische Fähigkeiten). Zusätzlich werden alle vier Wochen verschiedene biochemische Parameter bestimmt (Medikamentenspiegel, Katecholamine).

Das Hauptoutcomekriterium „Rückfall“ wurde vorab definiert als Zunahme im PANSS-Positiv-Score um mehr als zehn Punkte, ein CGIVeränderungswert von sechs oder mehr sowie eine Abnahme des GAFWertes um mehr als 20 Punkten (jeweils zwischen zwei Visiten).

\section{- Erste Ergebnisse}

Von Studienbeginn im November 2000 bis Rekrutierungsende im Juli 2004 wurden insgesamt $1393 \mathrm{~Pa}$ tienten gescreent, von denen 159 in die Langzeitstudie eingeschlossen werden konnten (131 nach Abschluss der Akutstudie und weitere 28 als Quereinsteiger). Davon haben bis dato im ersten Behandlungsjahr 105 Patienten (66\%) vorzeitig die Behandlung abgebrochen, 39 Patienten haben das erste Jahr erfolgreich beendet und 15 Patienten befinden sich momentan im ersten Behandlungsjahr. In das zweite Behandlungsjahr konnten bis Juli 200450 Patienten eingeschlossen werden (30 nach re- gulärer Beendigung des 1. Jahres und 20 über den Quereinstieg). Die Patienten waren bei Einschluss in die Langzeitstudie im Mittel 31,7 Jahre alt $(S D=10,2), 57 \%$ waren Männer.

Da die Studienmedikation erst nach Studienende im Juni 2006 entblindet wird, sind Aussagen zur differentiellen Wirksamkeit der Neuroleptika zum jetzigen Zeitpunkt noch nicht möglich. Dennoch lassen erste Auswertungen auf die generelle Wirksamkeit der Langzeitbehandlungsstrategien rückschließen. So war im ersten Behandlungsjahr bis dato lediglich ein Rezidiv nach den vorab definierten Kriterien zu beobachten (2,6\% von 39 Patienten, die das erste Behandlungsjahr erfolgreich abgeschlossen haben), klinisch bedeutsame Verschlechterung unterhalb der Rezidivkriterien waren ebenfalls seltene Ereignisse (z.B. Zunahme im PANSS-Positiv-Score um 7 Punkte bei ca. 8\%). Wie Abbildung 3 zeigt, ist bei Eintritt in die Langzeitstudie (im Mittel) noch leichte Positiv-Symptomatik vorhanden, die im weiteren Verlauf signifikant zurückgeht (LOCF-Analyse mit Fortschreibung der letzten Beobachtung bei Therapieabbruch; jeweils Vergleich der Werte bei Studieneintritt und nach einem Jahr mit dem Wilcoxon-Test). Der Rückgang an Negativ-Symptomatik sowie die $\mathrm{Zu}$ nahme im Funktionsniveau sind ebenfalls signifikant. 
Tab. 1 Liste der verwendeten Erhebungsinstrumente

\begin{tabular}{|c|c|c|}
\hline $\begin{array}{l}\text { Instrument } \\
\text { (Abkürzung) }\end{array}$ & $\begin{array}{l}\text { Instrument } \\
\text { (Volltext) }\end{array}$ & Autoren \\
\hline AIMS & $\begin{array}{l}\text { Abnormal Involuntary Movement Scale } \\
\text { (Abnorme unwillkürliche Bewegungen) }\end{array}$ & $\begin{array}{l}\text { Guy W. Washington DC: US Department of Health, } \\
\text { Education and Welfare, } 1976\end{array}$ \\
\hline CGI & $\begin{array}{l}\text { Clinical Global Impression } \\
\text { (Klinischer Gesamteindruck) }\end{array}$ & $\begin{array}{l}\text { Guy W. Bethesda MD: National Institute of Mental } \\
\text { Health, } 1976\end{array}$ \\
\hline CRS & Compliance Rating Scale & Kemp R, David A. BJD 1996; 169: 444-450 \\
\hline DAl & $\begin{array}{l}\text { Drug Attitude Inventory } \\
\text { (Einstellung gegenüber Medikamenten) }\end{array}$ & $\begin{array}{l}\text { Hogan TP et al. Psychological Medicine 1983; } \\
\text { 13: } 177-183\end{array}$ \\
\hline EPS & $\begin{array}{l}\text { Rating Scale for Extrapyramidal Side Effects } \\
\text { (Extrapyramidale Nebenwirkungen) }\end{array}$ & $\begin{array}{l}\text { Simpson GM, Angus JW. Acta Psychiatr Scand } \\
\text { Suppl. 1970; 212: 11-19 }\end{array}$ \\
\hline FEF & Fragebogen zur Erfassung der emotionalen Familienatmosphäre & Feldmann R et al. Diagnostica 1995: 4 \\
\hline FSKN & Frankfurter Selbstkonzeptskalen & Deusinger IM. Goettingen: Hogrefe, 1986 \\
\hline GAF & $\begin{array}{l}\text { The Global Assessment of Functioning Scale } \\
\text { (Soziales Funktionsniveau) }\end{array}$ & $\begin{array}{l}\text { Frances A et al. Washington DC: } \\
\text { American Psychiatric Association, } 1994\end{array}$ \\
\hline HAS & $\begin{array}{l}\text { Hillside Akathisia Scale } \\
\text { (Akathisie) }\end{array}$ & $\begin{array}{l}\text { Fleischhacker WW et al. Psychopharmacol Bull, } \\
\text { 1989; 25(2): 222-226 }\end{array}$ \\
\hline ICD-10 & $\begin{array}{l}\text { International statistical classification of diseases and related } \\
\text { health problems ( } 10^{\text {th }} \text { Revision) } \\
\text { (Internationale Klassifikation der Krankheiten (10. Revision) }\end{array}$ & World Health Organization. Genf: WHO, 1992 \\
\hline LQLP & $\begin{array}{l}\text { Lancashire Quality of Life Profile } \\
\text { (Lebensqualität) }\end{array}$ & Oliver J. Soc Work Soc Sci Rev 1991; 3: 5-45 \\
\hline MEL & Münchner Ereignisliste & Maier-Diewald W et al. München: MPI, 1983 \\
\hline PANSS & $\begin{array}{l}\text { Positive and Negative Symptom Scale } \\
\text { (Positiv- und Negativ-Symptomatik) }\end{array}$ & Kay S et al. Schizophr Bull 1986; 13: 261 \\
\hline SCPS & $\begin{array}{l}\text { Strauss-Carpenter Prognosis Scale } \\
\text { (Prognose-Skala) }\end{array}$ & $\begin{array}{l}\text { Strauss JS, Carpenter WT. Arch Gen Psychiatry } \\
\text { 1972; 27: 739-746 }\end{array}$ \\
\hline SVF & Stressverarbeitungsfragebogen & Janke W et al. Göttingen: Hogrefe, 1985 \\
\hline UKU & $\begin{array}{l}\text { Udvalg for Kliniske Undersogelser Side Effect Rating Scale } \\
\text { (Nebenwirkungen) }\end{array}$ & $\begin{array}{l}\text { Scandinavian Society of Psychopharmacology } \\
\text { Committee of Clinical Investigations. Acta Psy- } \\
\text { chiatrica Scandinavica 1987; 334(Suppl.): 81-94 }\end{array}$ \\
\hline
\end{tabular}

Nebenwirkungen waren bei Studienbeginn und im weiteren Verlauf nur leicht ausgeprägt (mittlerer EPSWert unter 2 bei einem möglichen Wertebereich von 0 bis 40; mittlerer UKU-Wert unter 5 bei einem Wertebereich von 0 bis 156). Die Compliance dagegen war im Mittel mit Werten über sechs sehr hoch (Wertebereich 1 = nicht compliant bis 7 = maximal compliant), ähnlich wie die Einstellung gegenüber den Medikamenten, die im Mittel mit Werten über 20 sehr positiv war (Wertebereich $0-30$ ).

Wie beabsichtigt konnte die Neuroleptikadosis beider Präparate im Niedrigbereich gehalten werden. Bei Eintritt in die Langzeitstudie lag sie im Durchschnitt bei 3,6 mg/Tag $(\mathrm{SD}=2,4)$ und variierte im weiteren Verlauf nur leicht zwischen 4,4 $\mathrm{mg} /$ Tag und 2,0 mg/Tag.

Ein nicht erwartetes Ergebnis war die mit 66\% sehr hohe Drop-Out-
Quote. Häufigste Ursache für einen vorzeitigen Therapieabbruch lag v.a. patientenseitig in der im Studienverlauf abnehmenden Bereitschaft, an der Studie weiter teilzunehmen (vgl. Abb. 2). Ungefähr ein Viertel ist auf die Nebenwirkungen der neuroleptischen Behandlung zurückzuführen. Dabei kann auf Grund der bis Studienende im Juni 2005 weiterhin bestehenden Verblindung noch nicht bestimmt werden, ob es hierbei differentielle Medikamenteneffekte gibt. In weiteren $15 \%$ war der Abbruch begründet in einer unzureichenden Response v.a. in Bezug auf eine trotz Medikation persistierende (nicht tolerierbare) Positivsymptomatik. Neben einzelnen weiteren, eher studienbedingten Gründen wie v.a. Instabilität im affektiven Bereich trugen auch Wohnort- und Diagnosewechsel (v.a. zu schizo-affektiver oder organisch bedingter Psychose) zu der hohen Zahl an Studienabbrüchen bei.
Bereits auf Grund von bei Einschluss in die Langzeitstudie erhobenen Parametern ließen sich Prädiktoren für einen Therapieabbruch identifizieren; dabei wurden in univariaten Analysen signifikante Parameter gefunden wie Quereinstieg (keine direkte Übernahme aus der Akutstudie), keine Teilnahme an einer psychologischen Intervention oder eine niedrige Compliance, die in der multivariaten Analyse alle überlagert wurden durch ausgeprägtere (Positiv-, Negativ- und Allgemeine) Symptome sowie stärkere neurologische Nebenwirkungen.

Erste Ergebnisse aus dem zweiten Behandlungsjahr weisen tendenziell darauf hin, dass die Erhaltungstherapie der Intervalltherapie hinsichtlich der Rezidivprophylaxe überlegen ist. Angesichts der bis dato zu geringen Fallzahlen müssen endgültige Aussagen jedoch noch abgewartet werden. 


\section{Diskussion}

Auch wenn auf Grund der weiterhin bestehenden Medikamentenverblindung noch keine Aussagen zu der eigentlichen Fragestellung der differentiellen Wirksamkeit von typischer vs. atypischer Neuroleptikabehandlung gemacht werden können, belegen die ersten Ergebnisse der in der Studie verbliebenen Patienten die Effektivität beider Präparate. Die Rückfallrate ist mit unter $3 \%$ sehr niedrig und vergleichbar mit der unter Depotmedikation (0\%) (11). Auch klinisch bedeutsame Verschlechterungen sind mit unter $10 \%$ sehr selten und eine anfangs noch leicht bestehende Positiv-Symptomatik remittiert nahezu vollständig auch bei niedriger Dosierung. Die Behandlung anderer (Negativ-) Symptome scheint ebenfalls effektiv zu sein, bei (im Mittel) nur geringen Nebenwirkungen. Auffallend ist die hohe Drop-OutQuote; zwei Drittel der Patienten nach schizophrener Erstmanifestation brechen die Langzeitbehandlung ab. Auch wenn zu vermuten ist, dass studientechnische Gründe mit dazu beitragen, scheint die Bereitschaft vieler Patienten eingeschränkt, eine Behandlung und Betreuung über einen längeren Zeitraum aufrecht zu erhalten. Dies macht deutlich, dass für ersterkrankte schizophrene Patienten spezielle Programme angezeigt sind, um sie in einer effektiven Langzeittherapie zu halten. Solche Programme werden nach Studienende in einer neu beginnenden Förderperiode entwickelt.

\section{Optimization of long-term} treatment strategies in first episode patients with schizophrenia Over the last decades, a set of effective bio-psycho-social long-term treatment strategies for schizophrenia have been developed. In the scope of the German Research Network on Schizophrenia, a multicenter study is currently under way, to optimize long-term treatment in first episode patients by testing two main hypotheses: are there differences in relapse rates regarding a) one year maintenance treatment with low-dose typical vs. atypical neuroleptic treatment and b) afterwards further maintenance treatment vs. drug discontinuation, both supplemented by prodrome based early intervention. First results of the ongoing study suggest a relapse preventing efficacy for both neuroleptics, whereas treatment non-adherence is quite often. Accordingly, special programs need to be developed to enhance treatment adherence.

\section{Key words}

First episode schizophrenia - longterm treatment - typical and atypical neuroleptics - maintenance treatment - intermittent treatment

\section{Literatur}

1. American Psychiatric Association. Practice guidelines for the treatment of patients with schizophrenia. Am J Psychiatry 1997; 154 (suppl 4): 1-63

2. American Psychiatric Association. Quick reference to the American Psychiatric Association Practice Guidelines for the treatment of psychiatric disorders: compendium 2002. American Psychiatric Publishing: Arlington, 2002

3. Csernansky JG, Mahmoud R, Brenner R. A comparison of risperidone and haloperidol for the prevention of relapse in patients with schizophrenia. N Engl J Med 2002; 346 $16-22$

4. Davis JM, Chen N, Glick ID. A meta-analysis of the efficacy of second-generation antipsychotics. Arch Gen Psych 2003; 60: 553-564

5. Deutsche Gesellschaft für Psychiatrie, Psychotherapie und Nervenheilkunde (DG PPN) (Ed.). Behandlungsleitlinie Schizophrenie. Darmstadt, Steinkoppf 1998

6. Emsley R. Risperidone in the treatment of first-episode psychotic patients: a double-blind multicenter study. Schizophr Bull 1999; 25: 721-729

7. Gaebel $W$, Jänner $M$, Frommann $N$, Pietzcker A, Köpcke W, Linden M, Müller P, Müller-Spahn F, Tegeler J. First vs. multiple episode schizophrenia: Two-year outcome of intermittent and maintenance medication strategies. Schizophr Res 2002; 53 145-159

8. Gaebel W, Janssen B, Riesbeck M. Modern treatment concepts in schizophrenia. Pharmacopsychiatry 2003; 36 (Suppl 3): 168-175

9. Gaebel W, Möller HJ, Buchkremer G, Ohmann C, Riesbeck M, Wölwer W, von Wilmsdorff $M$, Bottlender R, Klingberg S. Pharmacological long-term treatment strategies in first episode schizophrenia. Study design and preliminary results of an ongoing RCT within the German Research Network on Schizophrenia. European Archives of Psychiatry and Clinical Neuroscience (im Druck)

10. Geddes J, Freemantle N, Harrison P, Bebbington P. Atypical antipsychotics in the treatment of schizophrenia: systematic overview and meta-regression analysis. BM] 2000; 32: 1371-1376

11. Kane JM, Rifkin A, Quitkin F, Nayak D,
Ramos-Lorenzi J. Fluphenazine vs placebo in patients with remitted, acute first-episode schizophrenia. Arch Gen Psychiatry 1982; 21: 82-86

12. Kane JM, Leucht S, Carpenter D, Docherty JP. The expert consensus guideline series: optimizing pharmacologic treatment of psychotic disorders. J Clin Psychiatry 2003; 64 (Suppl 12): 1-100

13. Lehman AF, Steinwachs DM and the CoInvestigators of the PORT-Project. At issue: translating research into practice: the Schizophrenia Patient Outcomes Research Team (PORT) treatment recommendations. Schizophr Bull 1998; 24: 1-10

14. Leucht S, Pitschel-Walz G, Abraham D, Kissling W. Efficacy and extrapyramidal side-effects of the new antipsychotics olanzapine, quetiapine, risperidone, and sertindole compared to conventional antipsychotics and placebo. A meta-analysis of randomized controlled trials. Schizophr Res 1999; 35: 51-68

15. Leucht S, Barnes TR, Kissling W, Engel RR, Correll C, Kane JM. Relapse prevention in schizophrenia with new-generation antipsychotics: a systematic review and exploratory meta-analysis of randomized, controlled trials. Am J Psychiatry 2003; 160: 1209-1222

16. Leucht S, Wahlbeck K, Hamann J, Kissling $W$. New generation antipsychotics versus low-potency conventional antipsychotics: a systematic review and meta-analysis. Lancet 2003; 361: 1581-1589

17. Marder RS, Essock SM, Miller AL, Buchanan RW, Davis JM, Kane JM, Lieberman J, Schooler NR. The Mount Sinai Conference on the Pharmacotherapy of Schizophrenia. Schizophr Bull 2002; 28: 5-16

18. McEvoy J, Scheifler P, Frances A. The expert consensus quideline series: Treatment of schizophrenia. J Clin Psychiatry 1999; 60 (suppl 11): 3-80

19. National Institute for Clinical Excellence (NICE). Guidance on the use of newer (atypical) antipsychotic drugs for the treatment of schizophrenia. Technology Appraisal Guidance: 43 (www.nice.org.uk) 2002

20. Sanger TM, Lieberman JA, Tohen M, Grundy S, Beasley C, Tollefson G. Olanzapine versus haloperidol treatment in first-episode psychosis. Am J Psychiatry 1999;156: 79-87

\section{Korrespondenzsdresse:}

Prof. Dr. Wolfgang Gaebel

Klinik und Poliklinik für Psychiatrie

und Psychotherapie der Heinrich-Heine-

Universität Düsseldorf

Rheinische Kliniken Düsseldorf

Bergische Landstraße 2

40629 Düsseldorf

wolfgang.gaebel@uni-duesseldorf.de

Diese Publikation wurde im Rahmen des Kompetenznetzes Schizophrenie erstellt und vom Bundesministerium für Bildung und Forschung (BMBF) gefördert (Kennzeichen: 01 Gl 0232). 